\title{
Fibre-optic network architectures for on-board digital avionics signal distribution
}

\author{
Mohammad F. Alam ${ }^{1}$, Mohammed Atiquzzaman ${ }^{2, *, \dagger}$, Bradley D. Duncan ${ }^{3}$, \\ Hung Nguyen ${ }^{4}$ and Richard Kunath ${ }^{4}$ \\ ${ }^{1}$ Lucent Technologies, 555 Union Boulevard, Allentown, PA 18109-3286, U.S.A. \\ ${ }^{2}$ School of Computer Science, University of Oklahoma, Norman, OK 73019-6151, U.S.A. \\ ${ }^{3}$ Department of Electrical \& Computer Engineering, University of Dayton, Dayton, OH 45469-0226, U.S.A. \\ ${ }^{4}$ NASA Glenn Research Center, 21000 Brook Park Road, Cleveland, OH 44135, U.S.A.
}

\begin{abstract}
SUMMARY
Continued progress in both civil and military radio-frequency (RF) digital avionics applications is overstressing the capabilities and reliability of existing RF communication networks based on coaxial cables on board modern aircrafts. Future avionics systems will require high-bandwidth on-board communication links that are lightweight, immune to electromagnetic interference, and highly reliable. Fibre-optic networks can meet all these challenges in a cost-effective manner. Recently, on-board fibreoptic communication systems, where a fibre-optic network acts like a local area network (LAN) for digital data communications, have become a topic of extensive research and development. However, modern digital avionics systems require a system capable of transporting microwave and millimeter-wave RF signals that carry digital data on board an aircraft. Such optical networks transporting RF signals are completely different from the digital fibre-optic communication systems.

Recent advances in optoelectronics technology, including linear optoelectronic devices and wavelength division multiplexing (WDM), have opened a number of possibilities for designing on-board fibre-optic networks, including all-optical networks for digital avionics RF signal communication. The objective of this paper is to present a number of optical network architectures for transmitting RF signals carrying digital data in an aircraft.

In this paper, we investigate a number of different approaches, including all optical architectures, for building an optical network using fibre-optic transmission of on-board VHF and UHF RF signals. The relative merits and demerits of the network architectures are discussed, and the suitability of the architectures for particular applications is presented. Copyright (C) 2002 John Wiley \& Sons, Ltd.
\end{abstract}

KEY WORDS: fibre-optic communications; avionics signal distribution; aircraft signal distribution; radar signal distribution; optical network design

\footnotetext{
*Correspondence to: Mohammed Atiquzzaman, School of Computer Science, University of Oklahoma, Norman, OK 73019-6151, U.S.A.

†E-mail: atiq@ou.edu
}

Contract/grant sponsor: NASA; contract/grant number: NCC3-703

Published online 15 January 2002

Received 15 March 2001

Copyright (C) 2002 John Wiley \& Sons, Ltd. 


\section{INTRODUCTION}

Modern civil and military aircrafts require high-bandwidth data communication networks for on-board avionics systems. The communications requirements of these avionics systems can be broadly divided into two categories: in-flight communication, where communications originate and terminate at different systems on-board the aircraft, and remote communication, where communications take place between an on-board system and a system external to the aircraft, which may be a ground station, a satellite or another aircraft. Remote communications between an on-board system and a system external to the aircraft takes place on microwave and millimeter-wave radio-frequency (RF) communications channels, and a separate RF communication network exists on-board aircrafts for this purpose. Current and future advances in digital data communication between an aircraft and the outside world will require highbandwidth on-board RF communication networks. RF systems, with their interconnection network based on coaxial cables and waveguides, increase the complexity of the communication network on board modern civil and military aircrafts, with safety and reliability concerns due to electromagnetic interference (EMI) between the RF communication systems. A simple, reliable, and lightweight network that is free from the effects of EMI, and capable of supporting the broadband RF communications needs of the future on-board digital avionics systems cannot be easily implemented using existing coaxial cable-based systems. Fibre-optic communication systems can meet all the above challenges of modern avionics applications in an efficient and cost-effective manner [1]. In fact, a number of optoelectronic components for airborne applications, capable of withstanding the adverse environmental conditions on-board an aircraft, have already been developed [2-10]. The goal of this paper is to develop optical network architectures, which are simple, reliable, lightweight and capable of broadband communications, and capable of carrying RF signals in aircrafts.

Presently, two different optical wavelength regions are used in modern optical networks. These wavelength regions are around 1.3 and $1.55 \mu \mathrm{m}$ (1300 and $1550 \mathrm{~nm}$, respectively). For both of these wavelengths two methods can be used for fibre-optic transmission: analog and digital. Analog signals are continuously varying signals where the exact waveform of the RF signal needs to be preserved when transmitted over a fibre-optic link. The analog fibre-optic systems are also called linear fibre-optic systems. The linear fibre-optic transmitter and the linear fibre-optic receiver should be extremely stable, linear, and should have a large dynamic range. These stringent requirements increase the cost of linear (or analog) fibre-optic transmitters and receivers considerably. On the other hand, digital signals are composed of ones and zeros, and do not require preserving the exact waveform of the transmitted signal as long as the transmitted signal is not distorted to the extent that ones and zeroes cannot be distinguished from each other. For these reasons, digital fibre-optic communication equipment can perform well even when noise and distortion of the optical signal is present.

Fibre-optic networks onboard aircrafts for digital data communication have recently become an active area of research and development [11-15], and on-board digital fibre-optic communications systems have been successfully deployed on modern commercial aircrafts $[16,17]$. However, these digital networks are not capable of transporting avionics $R F$ signals on board an aircraft. Linear fibre-optic links can be employed to transport microwave and millimeter-wave RF signals on board an aircraft. These linear fibre-optic links take RF signals as input, transport them over fibre, and reproduce at the output an exact replica of the RF waveform fed to it at the input end. The RF signal itself may be modulated by a baseband signal 
by any of the analog or digital modulation techniques, but a linear fibre-optic link transmits the RF signal in the same manner irrespective of the modulation technique used for modulating the RF signal (by the baseband signal).

An optical transmitter launches the optical signal into an optical fibre. At the other end of the fibre, an optical receiver converts the optical signal back to RF. In most cases, the source or the destination of an RF signal is an antenna. Usually a single fibre can carry information in one direction only (simplex) which means that two fibres are required for bi-directional (duplex) communication. However, recent advances in wavelength division multiplexing (WDM) make it possible to use the same fibre for duplex communication using different wavelengths. Figure 1 shows a typical arrangement for RF signal transport over a linear fibre-optic link.

The objective of this paper is to present a number of optical network architectures for onboard RF signal distribution. The architectures use recently developed optical components for the all-optical networks in addition to the traditional hybrid RF-optical approach. It should be stressed that such optical networks for transporting $R F$ signals in aircrafts are completely different from the digital fibre-optic communication systems. To the best of our knowledge, application of fibre-optic networks for airborne RF signal distribution has not been reported in the literature. Due to the emergence of a number of digital avionics equipment requiring high-bandwidth connectivity, fibre-optic RF networks on board modern aircrafts will play a vital role in ensuring a low-noise and highly reliable RF communication system. In addition, efforts are already underway to integrate different communication networks onboard aircrafts into a single system [18], and the fibre-optic network architectures discussed in this paper can be vital in integrating both RF and digital in-flight communication systems on to a single infrastructure. Such integrated networks will reduce weight of an aircraft.

The rest of the paper is organized as follows. In Section 2, we introduce the principles of some of the emerging fibre-optic communication techniques based on recent advances in fibre-optic systems that can be applied to build optical networks for on-board signal and data distribution. Section 3 discusses fibre-optic single-source multiple-destination distribution networks, and

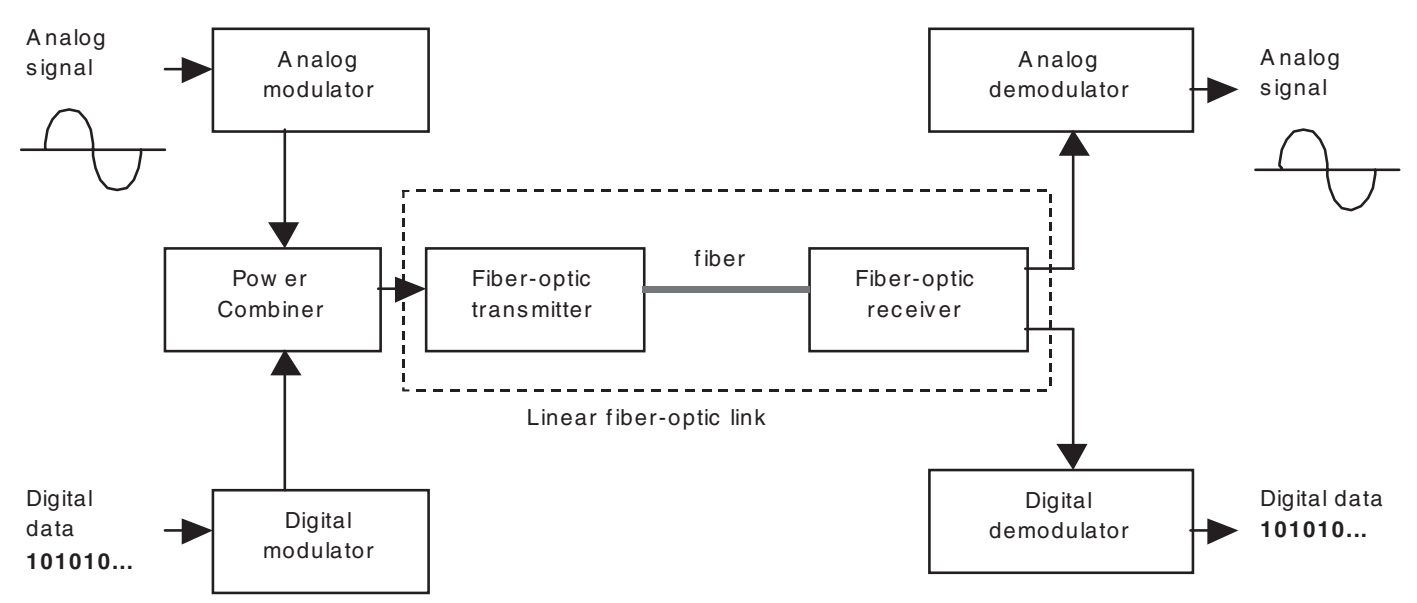

Figure 1. A linear fibre-optic link transports RF signals where the RF signal may be modulated by an analog or a digital modulation technique. 
Section 4 describes fibre-optic architectures for multiple-source multiple-destination networks. We conclude this paper in Section 5.

\section{BASIC CONCEPTS IN FIBRE-OPTIC RF SYSTEMS}

We first show how a simple RF system can be implemented using three different approaches. Figure 2 shows an RF system where an on-board VHF system having four channels in the 100 $150 \mathrm{MHz}$ frequency range communicates with a VHF antenna, and an on-board UHF system having 24 channels in the $950-1450 \mathrm{MHz}$ frequency range communicates with a UHF antenna. In the following subsections, we show how the RF system can be implemented using three different optical network based architectures. The systems referred to above could consist of navigational systems, atmospheric systems, fire and smoke alarm systems, ground communication systems, etc.

\subsection{Traditional approach}

Figure 3 shows the traditional fibre-optic approach to implement the RF communication system in Figure 2. Separate optical fibres are used for each point-to-point link. Thus, one pair of fibre connects UHF antenna 1 with UHF system 1, and another pair connects VHF antenna 2 with VHF system 2. Each point-to-point link requires a fibre-optic transmitter (Tx), a fibre-optic receiver $(\mathrm{Rx})$, and a continuous fibre-optic light path from each transmitter to the corresponding receiver. This system requires three cable segments: the left segment requires a cable with two fibres, the middle segment requires a cable with four fibres, and the right segment

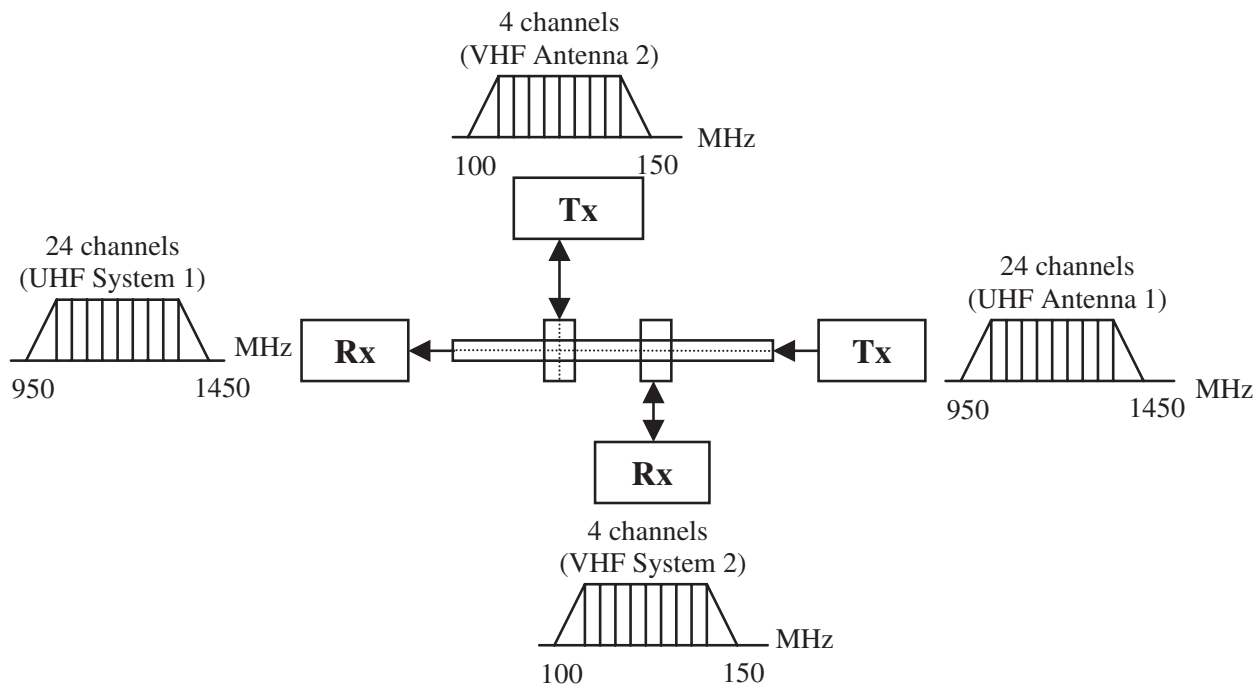

Figure 2. A simple fibre-optic communication system. The VHF antenna communicates with the VHF system, and the UHF antenna communicates with the UHF system. Rx represents an optical receiver, and Tx represents an optical transmitter. 


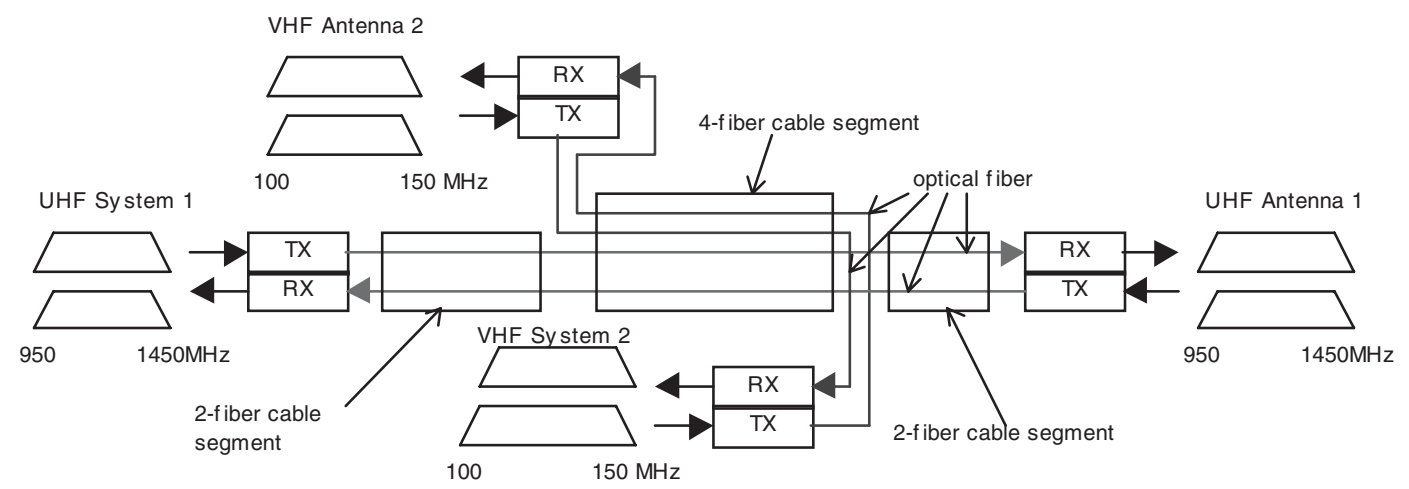

Figure 3. Traditional fibre-optic network solution to the interconnection problem of Figure 2. RX represents fibre-optic transmitters, and TX represents fibre-optic receivers.

requires two fibres. For the two fibres connecting the UHF antenna 1 with UHF system 1, splicing is required at every cable junction: once at the junction between the left and the central cable segments, and again at the junction between the central and the right cable segments.

This architecture is simple, economical and easy to implement when few antennas and systems need to be interconnected. However, the number of antennas and systems in future aircrafts will increase to provide higher safety and reliability. As the number of antennas and systems that need to be connected becomes higher and complex, it becomes almost impossible to use fibre cables with multiple fibres. If cables with multiple fibres are used, then they have to be spliced at many locations, making the system lossy and unreliable. On the other hand, using many singlefibre or twin-fibre cables reduces the advantage of weight reduction (essential for networks to be used in aircrafts) that could be obtained if fibre cables with multiple fibres could be used. One solution to the problem of signal loss due to splicing is to use a fibre-optic receiver and a transmitter at each node where a fibre has to be spliced. This second solution, which we call the hybrid RF-optical network, is discussed in Section 2.2. Another modern approach, where a number of different wavelengths are carried by an all-optical network using the WDM technology is discussed in Section 2.3.

\subsection{Hybrid RF-optical network}

The hybrid RF-optical network is shown in Figure 4. A single fibre carries all the RF signals from left to right, and another fibre carries all the RF signals in the opposite direction. These fibres are, however, split at every node where an RF channel needs to be added (or extracted) to (or from) the fibre. In addition to fibre-optic transmitters and receivers, we also need RF power combiners and RF demultiplexers. RF power combiners multiplex a number of different RF channels onto a single RF transmission line, while the RF demultiplexer extracts an RF channel from a band of RF frequencies. Each transmitting/receiving node must have a channel add-drop subsystem, which, for the simple network of Figure 2, requires only two add-drop subsystems for VHF antenna 2 and VHF system 2.

The advantage of this system is that a single pair of fibre, running from the nose to the tail of the aircraft, acts as the fibre-optic backbone. At any point in the aircraft, if a new RF channel 


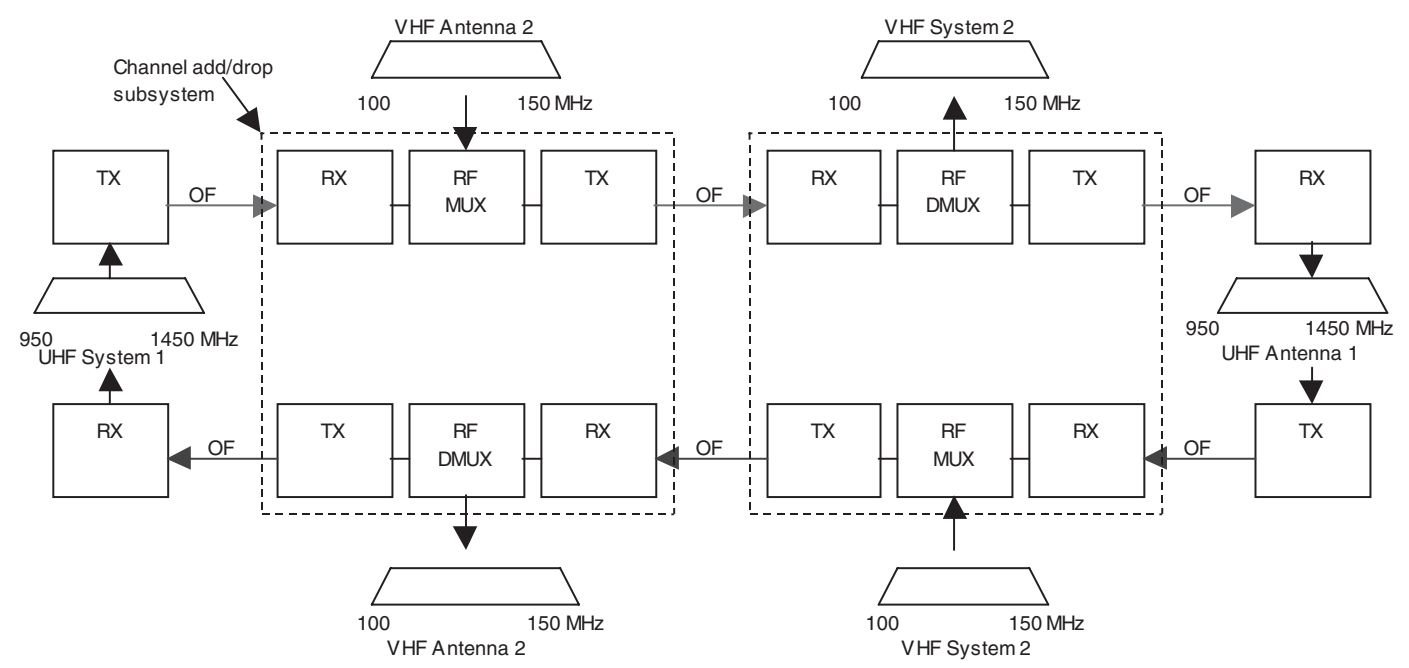

Figure 4. Hybrid RF-optical approach for Figure 2. OF represents optical fibre. MUX represents RF power combiner (multiplexer). DMUX represents RF demultiplexer. TX represents fibre-optic transmitter and RX represents fibre-optic receiver.

needs to be added to the fibre-optic backbone, the channels coming from the upstream fibre are converted to RF by an optical receiver, the local RF signal is multiplexed with the channels from upstream, and the multiplexed RF signal is again sent out to the downstream optical fibre using an optical transmitter. Although this approach saves fibre as compared to the traditional approach discussed in Section 2.1, additional fibre-optic transmitters and receivers, as well as RF power combiners and demultiplexers increase weight, power consumption, and signal interference of the system.

\subsection{All-optical approach}

WDM, which consists of sending more than one wavelength through an optical fibre, is a new and evolving technology. WDM technology allows sharing of a single fibre for simultaneously carrying two or more optical channels at different optical wavelengths. Thus, a single fibre carries a number of optical wavelengths, with each of the wavelengths carrying a number of multiplexed RF channels.

Figure 5 shows an all-optical network to support the communication needs of the RF system described in Figure 2. The network uses optical power combiners to multiplex a number of optical signals (carrying different RF channels) onto a single fibre. Optical power splitters split the optical power received from an incoming optical fibre into two or more equal or unequal fractions, and each fraction is transmitted to a new outgoing fibre. Both a splitter and a combiner have the same physical construction. So, a single component can work either as a combiner or as a splitter depending on the way it is connected. A wavelength demultiplexer filters out an optical wavelength to extract the required RF channel.

To map the radio frequencies to optical wavelengths, we choose 1330 and $1280 \mathrm{~nm}$ for the UHF and VHF systems, respectively. We still require two fibres, one for transmission in each 


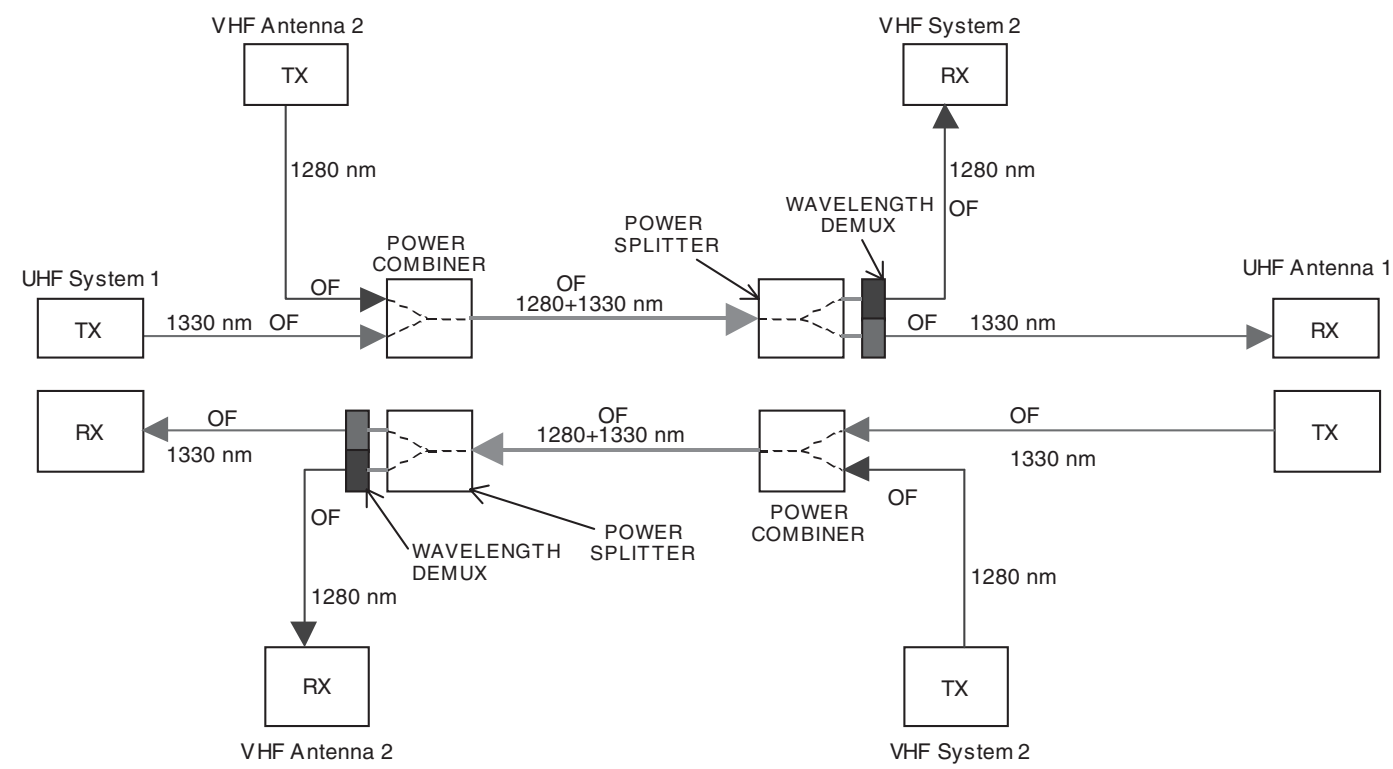

Figure 5. All-optical solution to the problem of Figure 2 using WDM. TX represents optical transmitter and RX represents optical receiver. OF represents optical fibre. Wavelength demultiplexer (DEMUX) separates two or more wavelengths from a combination of wavelengths.

direction. However, we do not need four fibres in the central segment as in Figure 2. For the fibre carrying signals from left to right, optical signals from two transmitters, one from UHF system 1 and the other from VHF antenna 2, are combined by an optical power combiner. One of the transmitters transmits at a wavelength $1280 \mathrm{~nm}$, while the other transmitter operates at $1330 \mathrm{~nm}$. The combiner at VHF antenna 2 combines the two wavelengths, and launches the combined power onto a single fibre that runs up to the point where VHF system 2 is located. At that point, a power splitter splits the total power into two equal halves.

The receiver for the VHF system 2 at $1280 \mathrm{~nm}$ will receive its channel from VHF antenna 2 via one of the output ports of the splitter. The other port of the splitter transmits half of the signal, which is received by UHF antenna 1 . The receiver at UHF antenna 1 receives its channel from UHF system 1 on $1330 \mathrm{~nm}$. VHF system 2 and UHF antenna 1 should receive their respective channels from the optical signal. Optical wavelength demultiplexers are used to filter out the optical wavelengths carrying the required RF channels. Communication in the reverse direction requires another fibre running in the opposite direction, and two more combiners/splitters.

The advantage of this method is that it is an all-optical solution where an optical signal, injected into the system by an optical transmitter, remains in optical form until the optical signal is received by the corresponding optical receiver. Thus, a complete light-path exists between an optical transmitter and an optical receiver for every wavelength that is present in the system. There is no optical-to-RF and RF-to-optical conversion (as in the solution of Section 2.2), which may degrade the signal-to-noise ratio of the RF signal being carried by the optical signal. Moreover, the ability to carry many RF signals on different wavelengths over the same fibre results in savings in weight, which is crucial for networks to be used in aircrafts. In addition, the 
use of wavelength multiplexers and demultiplexers, which are passive components requiring no power supply, reduces the power consumption when compared to hybrid RF-optical systems.

\section{FIBRE-OPTIC SIGNAL DISTRIBUTION SYSTEMS}

In this section, we discuss the problem of designing a fibre-optic network that distributes an RF signal (originating from a single source such as an antenna) to a number of systems on an aircraft. Figure 6 shows the distribution system design problem. Here, a single VHF antenna receives RF signals that need to be distributed to a number of on-board systems $(1,2,3$, etc.). We can adopt either a hybrid RF-optical approach introduced in Section 2.2, or an all-optical approach, as explained in Section 2.3. In the following sections, we discuss both these approaches to implement the distribution system in Figure 6. Such distribution systems could be used to distribute entertainment, such as audio and video, in an aircraft.

\subsection{Hybrid RF-optical network}

In the hybrid RF-optical solution, we extend the idea of Section 2.2 to multiple nodes. There is only one pair of fibre running from the nose to the tail of an aircraft as shown in Figure 7. At each point (called a node) the incoming optical signal coming from the left side is converted to $\mathrm{RF}$, the local RF channel is extracted, and a new optical signal is transmitted by a fibre-optic transmitter to the outgoing fibre-optic link as shown in Figure 8.

This solution is simple and requires only a single fibre backbone that runs over the length of the aircraft. However, each node requires a fibre-optic transmitter and a receiver.

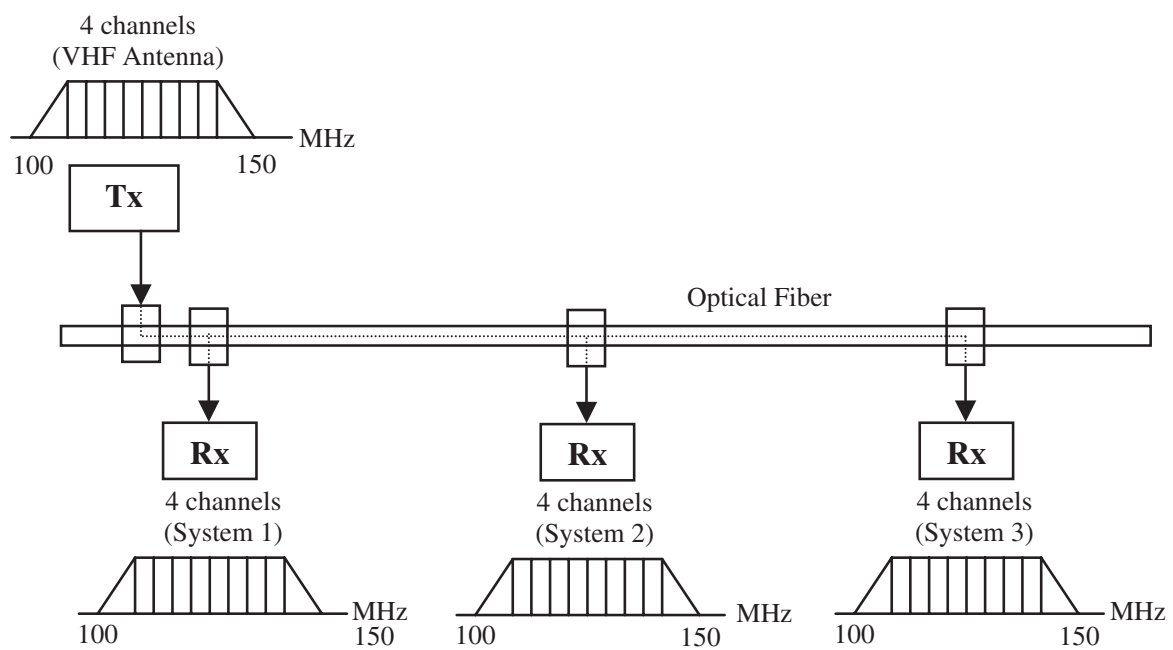

Figure 6. A fibre-optic signal distribution system. Tx represents fibre-optic transmitter and Rx represents fibre-optic receiver. 


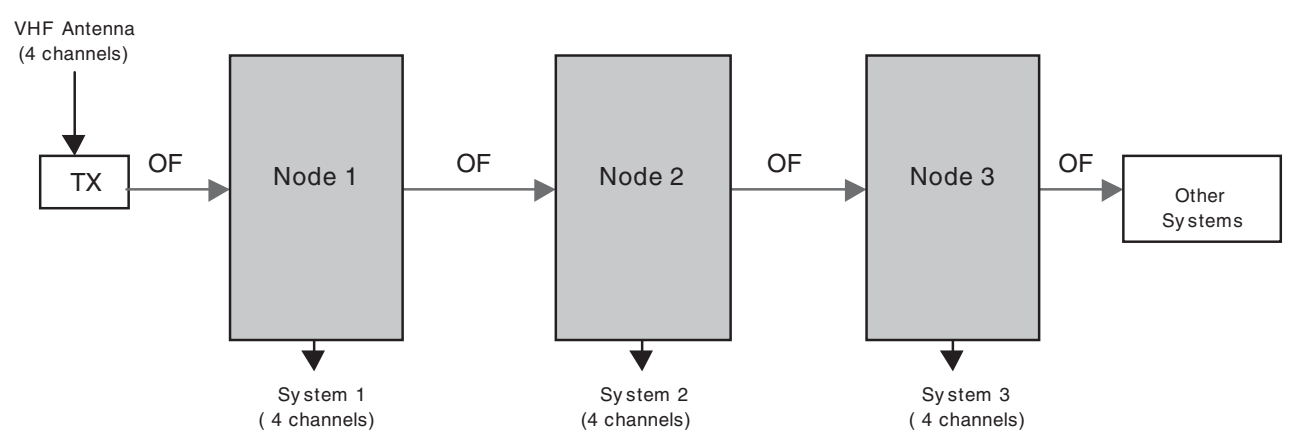

Figure 7. Schematic diagram of hybrid RF-optical solution of the problem in Figure 6. TX is a fibre-optic transmitter. OF is optical fibre.

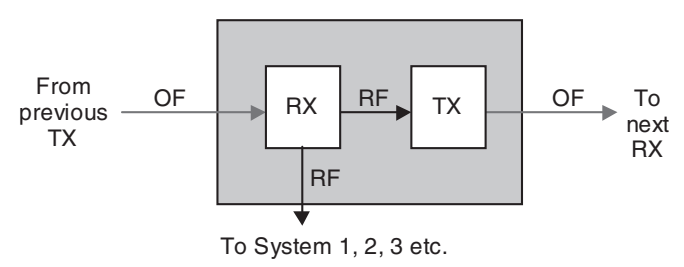

Figure 8. Detailed schematic of a receiving node in Figure 7. RX is a fibre-optic receiver, and TX is a fibreoptic transmitter. OF is optical fibre, and RF is radio-frequency cable.

\subsection{All-optical network}

A fibre-optical splitter is a device which splits the optical power received from a single fibre into a number of fractions, and then transmits each fraction on a separate fibre. It is usually a passive device, which is simple and lightweight in construction. Using one or more levels of splitters, it is possible to generate a distribution tree from a single source of fibre-optic signal.

Figure 9 shows the all-optical network solution to the signal distribution problem of Figure 6. In this approach, we first convert the RF signal into an optical signal using a fibre-optic transmitter. The optical signal is then split into eight by a one-to-eight optical splitter. Each of the optical signals may go through repeaters, where the weak optical signal is first converted to RF, and then re-transmitted by a fibre-optic transmitter, as in Figure 10. Each of these optical signals is then again split into eight by another level of optical power splitters. If repeaters are not used, then each receiver receives only $\frac{1}{64}$ of the optical power transmitted by the optical transmitter at the antenna.

This system is highly advantageous because the optical signal is distributed by an all-optical network (except for repeaters, if needed) and the overall design of the system is simple and easy to implement. 


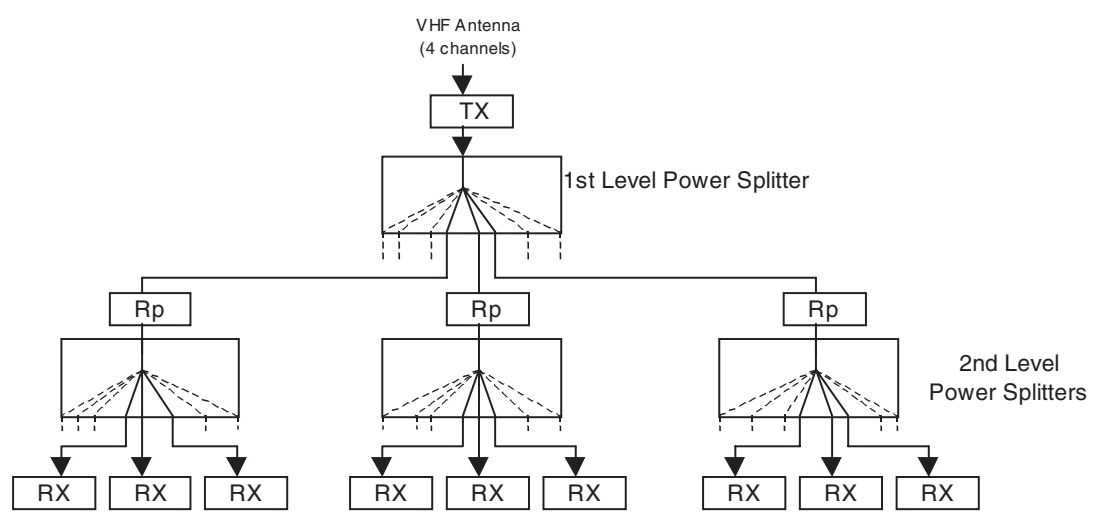

Figure 9. Schematic diagram of all-optical solution to the problem of Figure 6. There are two rows (levels) of power splitters. Not all branches are shown for clarity. TX represents fibre-optic transmitter, and RX represents fibre-optic receiver. Rp represents optional repeaters that may or may not be required depending on the signal strength at the final receivers. After the top TX, all signals are optical.

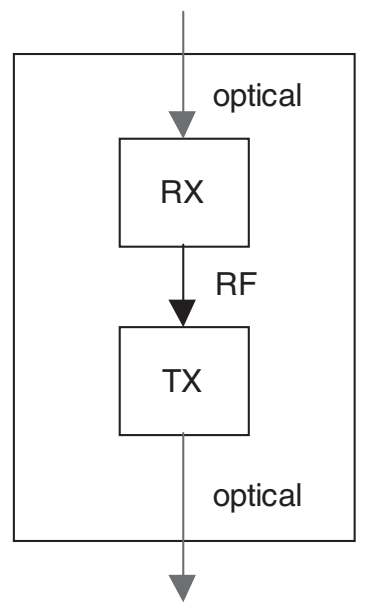

Figure 10. Detailed schematic of a repeater $(\mathrm{Rp})$ in Figure 9, which consists of a single fibre-optic receiver (RX) followed by a single fibre-optic transmitter (TX) to increase the strength of the optical signal.

\section{SYSTEMS WITH MULTIPLE TRANSMITTERS AND RECEIVERS}

Finally, we describe the design methodology for an on-board system where multiple nodes are transmitting, and each of the systems on board the aircraft must be capable of receiving the transmission from any one of the transmitters.

Figure 11 shows a communications architecture with three antennas from where optical signals are transmitted, and these signals should be transported to any of the on-board systems (three systems are shown in Figure 11). As in Section 3, we can take either the hybrid RF-optical approach, or the all-optical approach. 


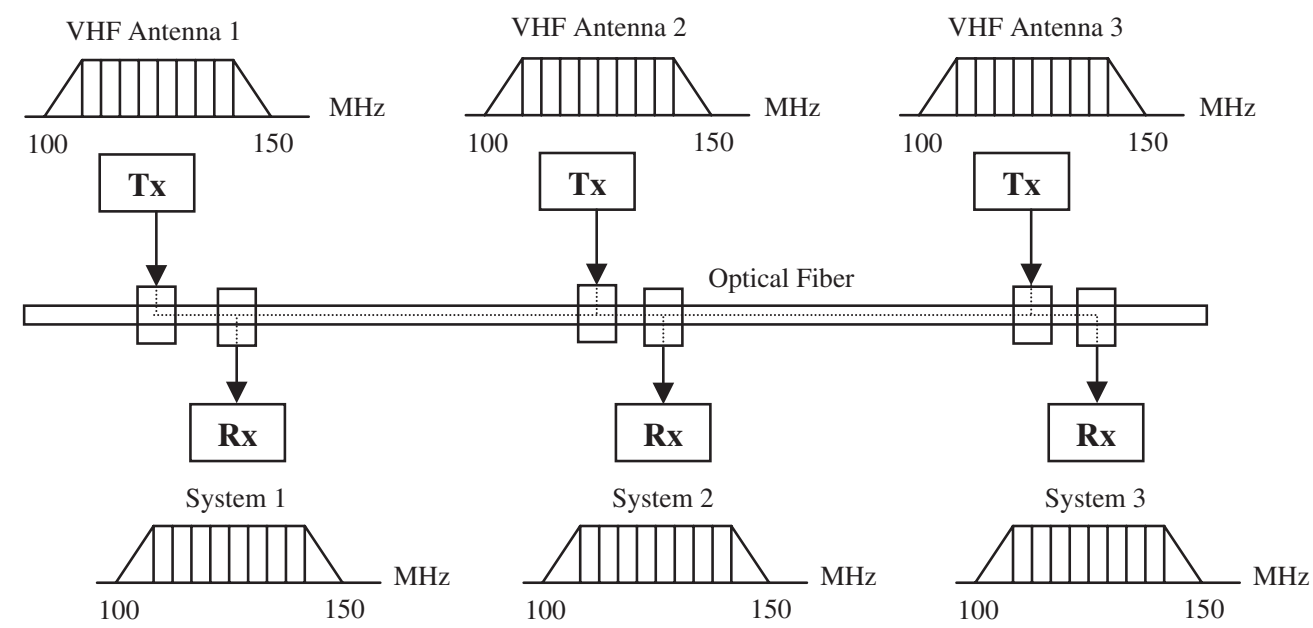

Figure 11. Fibre-optic communication network architecture with multiple transmitters and receivers.

\subsection{Hybrid RF-optical approach}

In this approach, we use two fibres running in opposite directions throughout the length of an aircraft. At the location of each (transmitting) antenna or (receiving) system, the optical signal is first converted to RF, and then either a new RF channel is added in case of a transmitting node, or the received signal is retransmitted in case of a receiving node. Figure 12 shows the block diagram of such a multiple-transmitter multiple-receiver system. The antenna nodes are transmitting $(\mathrm{Tx})$ nodes, while the system nodes are receiving $(\mathrm{Rx})$ nodes. The details of each of the transmitting and receiving nodes are shown in Figures 13 and 14, respectively. At each of the transmitting and receiving nodes, the optical signal is converted to RF, an RF channel is added or extracted, and then a new optical signal is transmitted from the RF signal.

This solution has the advantage that the optical signal is being amplified at each of the nodes thereby overcoming the signal loss problem at the connectors. In addition, this network utilizes only two counter-running fibres. However, this system has the disadvantage that many fibreoptic transmitters and receivers are required. Also, a signal that was injected to the fibre at the leftmost end reaches the rightmost end after a large number of optical-to-RF and RF-to-optical conversions, which may cause the RF signal to degrade considerably resulting in the RF signal having an unacceptable signal-to-noise ratio.

\subsection{All-optical approach}

In the all-optical approach, we use the coarse WDM technology, where a number of wavelengths, which are widely separated, are transmitted over the same fibre as shown in Figure 15. Different transmitters transmit optical signals at different wavelengths. Two counterrunning fibres carry optical signals in two directions. From each antenna, two separate transmitters are used to transmit the optical signals into the two fibres carrying signals into two (opposite) directions. Each receiving system receives optical signals from an antenna either from the upper fibre or the lower fibre, but not from both fibres from the same antenna (whether the 


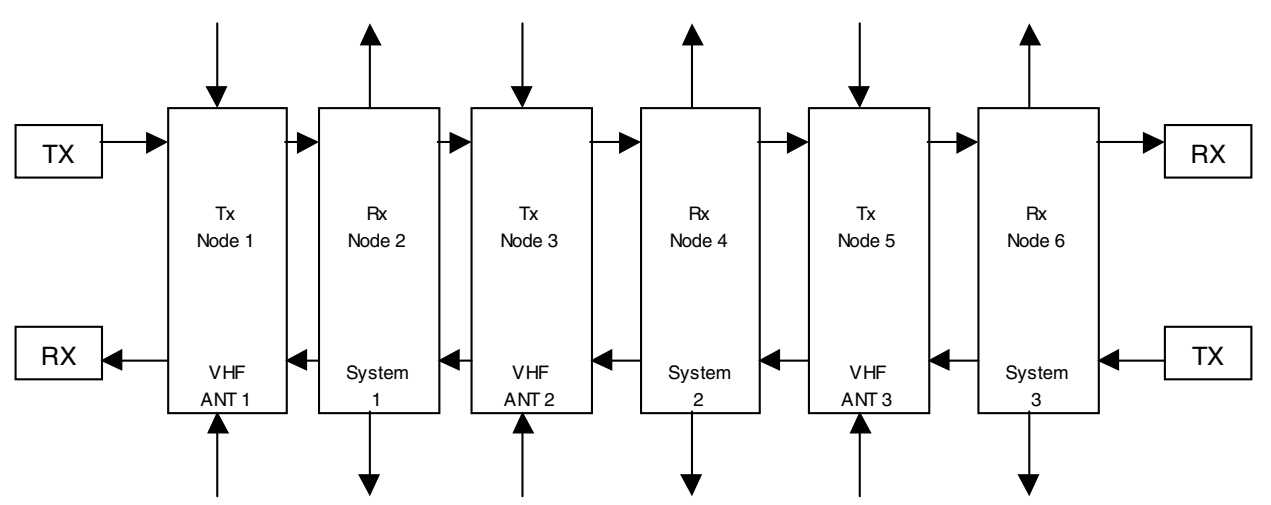

Figure 12. Hybrid RF-optical solution to multiple-source multiple-destination problem. TX is a fibre-optic transmitter and RX is a fibre-optic receiver. In this example, all antennas are transmitting (Tx) nodes, and all on-board systems are receiving $(\mathrm{Rx})$ nodes.

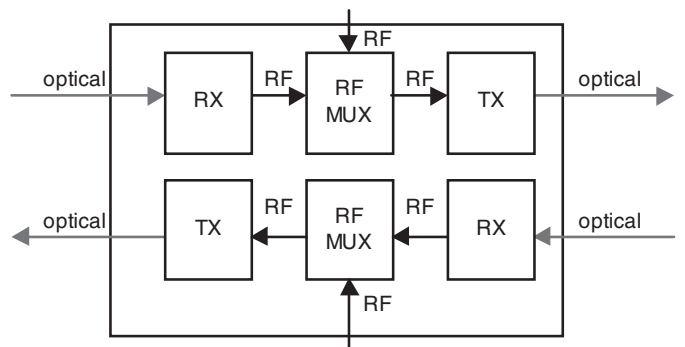

Figure 13. Detailed schematic diagram of a transmitting (Tx) node in Figure 12. In each direction, the optical signal is first converted to RF by a fibre-optic receiver (RX), and then a local RF channel is multiplexed with the received RF signal by and RF multiplexer (RF MUX). The combined RF signal is then converted to optical by a fibre-optic transmitter (TX).

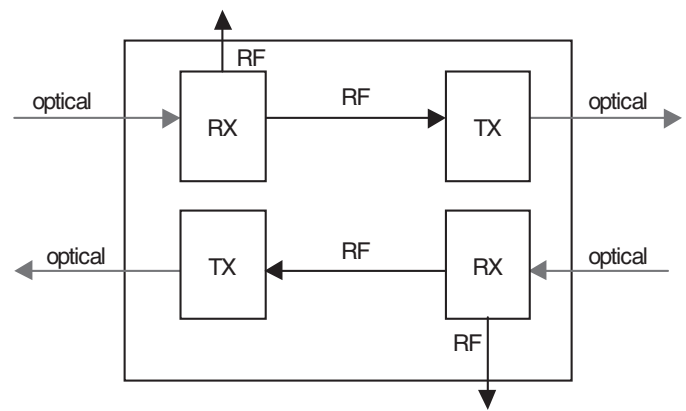

Figure 14. Detailed schematic of a receiving $(\mathrm{Rx})$ node in Figure 12. In each direction, the optical signal is converted to RF by a fibre-optic receiver (RX), and then the RF signal is re-transmitted by a fibre-optic transmitter (TX) in optical form. A fraction of the RF signal is also fed to the local RF receiver. 


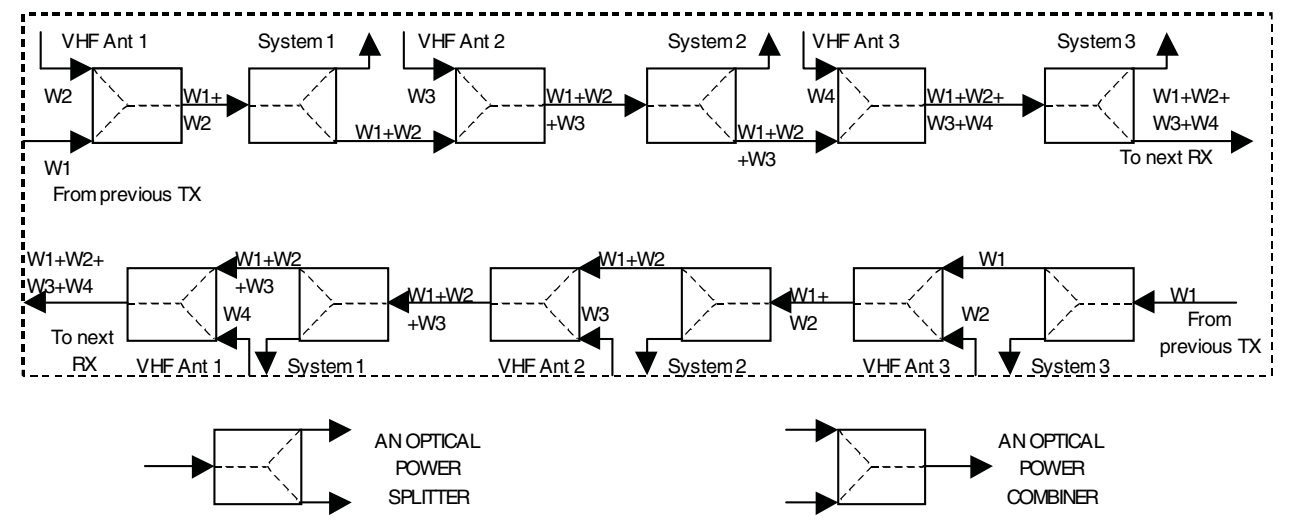

Figure 15. All-optical solution using dense wavelength division multiplexing for implementation of multiple-source multiple-receiver network architecture. The fibre-optic transmitters and receivers are not shown here for clarity. Each of the up-going arrows on the upper row as well as each of the down-going arrows on the lower row supply optical signal to a fibre-optic receiver in systems 1,2, 3, etc. Each receiver requires a wavelength demultiplexer to separate all the wavelengths present. Optical signals are transmitted from VHF antennas 1, 2, and 3 to optical power combiners.

receiving system is located to the left or to the right of the antenna determines whether the signal is received via the upper fibre or the lower fibre).

Each of the transmitting nodes adds a new wavelength $\left(\mathrm{W}_{1}, \mathrm{~W}_{2}\right.$, etc.) to the set of wavelengths being carried by the optical fibre with the help of an optical power combiner. Each receiving node uses an optical splitter to split the incoming signal and extracts a fraction of the optical signal being transmitted through the optical fibre. Each of the receiving systems receives more than one wavelength. By using wavelength demultiplexers, each of the wavelengths $\left(\mathrm{W}_{1}, \mathrm{~W}_{2}\right.$, $\mathrm{W}_{3}$, etc.) can be separated. From each of the separated wavelengths, separate receivers can retrieve the separate RF signals transmitted by different transmitting nodes (antennas).

This approach has the advantage that the system is lightweight due to the use of only two fibres to interconnect a large number of transmitting and receiving nodes. The solution is alloptical, and a light-path is established between every optical transmitter and receiver. Since there is no optical-to-RF and RF-to-optical conversion, the problem of signal-to-noise ratio degradation is eliminated which is a substantial problem in the hybrid RF-optical solution of Section 4.1. This approach, however, has a disadvantage that each signal is split many times and that can degrade the signal strength considerably. Also, WDM components tend to be costlier than single wavelength systems. By choosing proper power-splitting ratios at the power splitters, the signal strength may be kept at an acceptable level at each point despite the losses at the connectors and splitters. Since most of the components are passive, the weight and power requirement for this approach are also less than the hybrid approach of Section 4.1.

\section{CONCLUSION}

Fibre-optic communication systems that carry radio-frequency signals from one point to another are completely different from the ubiquitous digital fibre-optic communication systems 
in use today. These analog or linear fibre-optic systems carry microwave and millimeter-wave RF signals from one place to another without any distortion of the RF signal.

In this paper, we presented a number of different previously unexplored approaches for fibreoptic transmission of avionics RF signals using recently developed components. In almost all cases, we can either take a hybrid RF-optical approach or an all-optical approach. Reduced weight, lower electromagnetic interference, and lower power consumption are critical design issues for networks to be used in aircrafts.

The hybrid RF-optical approach has the advantage of low signal loss due to repeated regeneration of the RF signal, and requires less number of fibres. However, the use of too many fibre-optic transmitters and receivers increases the weight and cost of the hybrid system, and also increases EMI. The all-optical approach, on the other hand overcomes all these difficulties by ensuring a continuous light-path from a fibre-optic transmitter to one or more fibre-optic receivers while sustaining a strong optical signal. Also, an all-optical approach has the potential for replacing multiple-fibre cables by carrying a number of RF signals on different wavelengths on a single fibre simultaneously, thereby reducing the weight. In addition, all-optical solutions use a number of passive components like power combiners, power splitters and wavelength demultiplexers, all of which are passive devices requiring no power supply. Hence, an all-optical solution requires less power than the hybrid RF-optical solution. However, all-optical solutions tend to be expensive due to high cost of wavelength multiplexing and demultiplexing equipment.

\section{ACKNOWLEDGEMENTS}

This work is supported by NASA grant no. NCC3-703.

\section{REFERENCES}

1. Alam MF, Atiquzzaman M, Duncan B, Nguyen H, Kunath R. Fibre-optic network architectures for on-board radar and avionics signal distribution. IEEE Radar 2000 Conference, Washington, DC, 7-12 May, 2000.

2. Wilgus JS, Beranek MW, Chan EY, Hager HE, Le QN. Potential opportunities for utilizing commercial optoelectronics in avionics fibre-optic networks. AIAA/IEEE 18th Digital Avionics Systems Conference, St. Louis, MO, 24-29 October, 1999.

3. Zhang J, Ni Y, Zheng L. Military avionics optical fibre data buses with active coupler configurations. IEEE Aerospace and Electronic Systems Magazine 1999; 14(7):27-33.

4. Zhang J. Proposed airborne optical fibre asynchronous CDMA systems using fully optical orthogonal codes for realtime avionic data distributions and computer interconnects. IEEE Aerospace and Electronic Systems Magazine 1999; 14(7):35-42.

5. Bedoya CA. Fly-by-Light Advanced Systems Hardware (FLASH) program. In Proceedings of SPIE 1995; 2467: $2-13$.

6. Beranek MW, Chan EY, Hager HE, Le QN, Wilgus JS. Emerging opportunities for applying COTS optoelectronics in avionics fibre-optic networks. Microprocessors and Microsystems 1999; 22(8):439-451.

7. Beranek MW, Chan EY, Hager HE, Le QN. Status of optoelectronic module packaging for avionics/aerospace applications. In 1998 11th Annual Meeting, IEEE Lasers and Electro-Optics Society, Orlando, FL, U.S.A., 1-4 December, 1998; 329-330.

8. Horwitz D. COTS fibre optic interconnect solutions for mobile platforms. 48th Electronic Components \& Technology Conference, Seattle, WA, U.S.A., 25-28 May, 1998; 395-403.

9. Lubowe AG, Million TP, Sayegh EG, Baumann F. Multifibre optical connectors for avionics. Proceedings of SPIE 1996; 2840:14-22.

10. Chan EY, Beranek MW, Davido KW, Hager HE, Hong CS, St. Pierre RL. Challenges for developing low-cost avionics/aerospace-grade optoelectronic modules. In Proceedings of the Electronic Components \& Technology Conference, 1996; 1122-1129. 
11. Zhang J. High-speed avionic optical fibre CDMA networks. IEEE Aerospace and Electronic Systems Magazine 1999; 14(6): $15-21$.

12. Zhang J. Optical fibre data bus modulation techniques for military avionics. IEEE Aerospace and Electronic Systems Magazine 1999; 14(4):31-37.

13. Nelson GL, Rao NM, Krawczak JA, Stevens RC. Design process for a photonic network for military platforms. Proceedings of SPIE 1999; 3541:179-190.

14. Drogin EM, Leopardi AM. High speed data link concepts for military aircraft. National Aerospace Electronics Conference, Dayton, OH, U.S.A., 13-17 July, 1998; 136-143.

15. Halski D. Fly-by-light flight control systems. Aerospace Engineering 1996; 16(9):11-18.

16. Anderson DE, Beranek MW. 777 optical LAN technology review. Proceedings of the Electronics Components and Technology Conference, Seattle, WA, 25-28 May, 1998.

17. Kerr RB. Data communications management for the Boeing 777 aircraft. AIAA/IEEE 14th Digital Avionics Systems Conference, Cambridge, MA, 5-9 November, 1995.

18. Murdock JK, Koenig JR. Open systems avionics network to replace MIL-STD-1553. In Proceedings of the AIAA/ IEEE 19th Digital Avionics Systems Conference, Philadelphia, PA, 2000.

\section{AUTHORS’ BIOGRAPHIES}

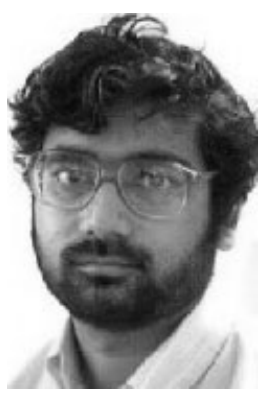

Mohammad Ferdous Alam received the BSc degree in electrical engineering from Bangladesh University of Engineering and Technology (BUET) in 1992. He taught at the electrical engineering department of BUET during 1992-95. Later, Mr Alam continued his graduate study at the School of Engineering of the University of Dayton in Ohio where he obtained his MS and PhD degrees in Electro-Optics in 1997 and 2000, respectively. His doctoral dissertation research involved studies on quality of service issues in next generation high-speed optical networks. He is currently a member of technical staff at Lucent Technologies Microelectronics Group in Allentown, Pennsylvania. His job duties include design and development of semiconductor integrated circuits for broadband communications applications.

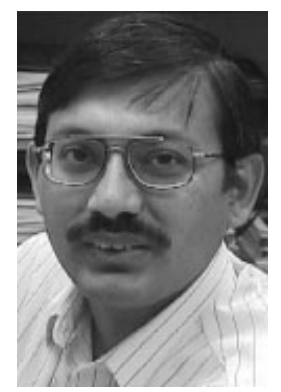

Mohammed Atiquzzaman received the MSc and $\mathrm{PhD}$ degrees in electrical engineering and electronics from the University of Manchester Institute of Science and Technology, England in 1984 and 1987, respectively. Currently he is faculty member in the School of Computer Science the University of Oklahoma, USA. He has served as senior editor of the IEEE Communications Magazine, and currently serves on the editorial boards of the Computer Communications journal, Telecommunications Systems journal and Real Time Imaging journal. He has guest edited special issues on many topics in various journals. He has also served in the technical program committee of many national and international conferences including IEEE INFOCOM, IEEE Globecom and IEEE Annual Conference on Local Computer Networks. He is a senior member of the IEEE. His current research interests are in Radio Frequency transmission over Optical Networks, Next Generation Internet, Broadband Networks, Multimedia, multiprocessor systems, and image processing. He has over 100 refereed publications in the above areas, most of which can be accessed at www.cs.ou.edu/ atiq/. He can be contacted at atiq@ou.edu 


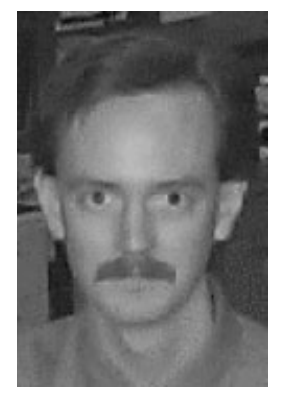

Bradley D. Duncan was born in 1963. He received the BSEE, MSEE and PhD degrees in Electrical Engineering from Virginia Tech in 1986, 1988, and 1991, respectively. Since the Fall of 1991 he has served on the Electrical \& Computer Engineering faculty at the University of Dayton, where he is currently a tenured Associate Professor. Dr Duncan also holds a joint appointment with the graduate Electro-Optics Program at the University of Dayton. His research interests focus on laser radar, fibre optic sensing and communications, integrated optical waveguide design, and optical non-destructive evaluation of advanced materials. He has published frequently in each of these areas. Dr Duncan is a Senior Member of the IEEE and is a member of both the OSA and SPIE. In 1998 Dr Duncan received the 'Engineering Best Professor of the Year' award from the engineering students at the University of Dayton.

Hung D. Nguyen is a research engineer for the Communications Technology Division of NASA Glenn Research Center, where he is engaged in the development of semiconductor integrated devices for highspeed communication systems. His research interests focus on novel thin-film materials, semi-conducting material characterization, optic and electronic packaging, polymer-based integrated optics, and microfabrication technology. Author of numerous technical papers on micro-lithography technology, Nguyen earned his $\mathrm{PhD}$ in electrical engineering and applied physics from Case Western Reserve University.

Richard R. Kunath, Jr. was born in Cleveland, Ohio in 1958. He received a BS and MS in Physics from Bowling Green State University in 1980 and 1982, respectively. Mr Kunath taught at BGSU until he joined the NASA Glenn Research Center in 1983. Mr Kunath's research interests have been advanced antenna designs for space communications applications, including MMIC-based phased array antennas and the use of photonics in phased array antenna systems. Mr Kunath is a member of AIAA, Sigma Pi Sigma, and has been a member of IEEE and SPIE. 